\title{
Practical Differential Diagnosis for CT and MRI
}

\author{
E.C. Lin, E.J. Escott, K.D. Garg, A.G. Bleicher, and D. Alexander, eds.
}

New York, NY: Thieme Medical Publishers, Inc., 2008, 350 pages, $\$ 54.95$

This soft-cover book concisely describes the identification of key radiologic signs with easy-to-use tables and bullet-point lists that facilitate rapid review of important findings, differential points, and disease entities. The book is organized into 7 sections. Section 1 (Brain) has 22 topics dealing with tumors, infarcts, infections such as HIV, and dense and calcified lesions. Section 2 (Head and Neck) discusses 20 topics, including various lesions: calvarial, skull base, sinonasal, orbital, salivary gland, thyroid, carotid space, congenital neck, lymph node, ear, temporal bone, and vocal cord. Cancers and radiation changes of the head and neck are also discussed. Section 3 (Chest) contains 24 topics, including various patterns such as ground-glass and tree-in-bud; feeding vessel signs; airway diseases such as bronchiectasis and emphysema; aortic aneurysm and dissection; and pulmonary diseases such as pneumonia, pneumoconiosis, nodules, and emboli. Section 4 (Abdomen) has 41 topics, including cystic, hemorrhagic, calcified, and diffuse hepatic lesions; cystic and vascular pancreatic lesions; adrenal adenoma and metastasis; hyperdense renal lesions; bowel imaging signs; diverticulitis; bowel cancers; peritoneal and retroperitoneal masses; calcification; and fibrosis. Section 5 (Pelvis) includes 10 topics, among which are uterine tumors, ovarian masses, periurethral cysts, and prostate cysts. Section 6 (Musculoskeletal) contains 23 topics, including bone and soft-tissue tumors, bone marrow edema, meniscal tears, and cruciate ligament and rotator cuff injuries. Section 7 (Spine) has 9 topics related to vertebral fracture and infection, spinal canal lesions, diskitis, and degenerative processes.

This book serves as a useful daily aid for the differential diagnosis of common and rare radiologic findings and conditions. For each finding and diagnosis, a complete list is provided to help clinicians differentiate between various diseases with similar imaging findings. This portable book is ideal for residents and fellows preparing for specialty board examinations. Unfortunately, there are no illustrations because the book is meant to be used while interpreting studies at the workstation or view box. The book is intended to be a handy reference for both trainees and practicing radiologists.

\author{
E. Edmund Kim \\ M.D. Anderson Cancer Center \\ 1515 Holcombe Blvd., Unit 1264 \\ Houston, TX 77030 \\ E-mail: ekim@di.mdacc.tmc.edu
}

